Bull. Chem. Soc. Ethiop. 2013, 27(2), 265-271.

Printed in Ethiopia

DOI: http://dx.doi.org/10.4314/bcse.v27i2.11

ISSN 1011-3924

(c) 2013 Chemical Society of Ethiopia

\title{
MICROWAVE-ASSISTED SYNTHESIS OF SOME 5(6)-NITRO-1H- BENZIMIDAZOLES AND THEIR HYDRAZIDE DERIVATIVES
}

\author{
Fatih Yılmaz ${ }^{1}$, Emre Menteşe ${ }^{1 *}$, Nesrin Karaali ${ }^{1}$ and Bahittin Kahveci ${ }^{2}$ \\ ${ }^{1}$ Department of Chemistry, Faculty of Arts and Sciences, Recep Tayyip Erdoğan University, \\ 53100, Rize, Turkey \\ ${ }^{2}$ Department of Nutrition and Dietetics, Faculty of Health Sciences, Karadeniz Technical \\ University, Trabzon, Turkey
}

(Received October 17, 2012; revised February 8, 2013)

\begin{abstract}
A series of 5(6)-nitro- $1 H$-benzimidazole and their hydrazide derivatives were synthesized starting from iminoester hydrochloride and 4-nitro- $o$-phenylenediamine under microwave irradiation. The structure of all synthesized compounds was confirmed by IR, ${ }^{1} \mathrm{H}-\mathrm{NMR},{ }^{13} \mathrm{C}-\mathrm{NMR}$, ESI-MS spectroscopy and elemental analysis.
\end{abstract}

KEY WORDS: Benzimidazole, Iminoester hydrochloride, Microwave irradiation, Hydrazine hydrate

\section{INTRODUCTION}

Benzimidazoles are crucial structures for medicinal chemistry because they show important biological and pharmaceutical activities including anti-cancer, anti-microbial, anti-fungal, antiulcer and anti-viral $[1,2]$. Furthermore, various kinds of benzimidazole derivatives are found in diverse array of compounds, including biologically and therapeutically active agents [3], natural products [4]. Therefore, these compounds have been attracted attention of synthetic organic chemist.

A number of synthetic methods have been developed in recent years to bring out new reagents for the synthesis of 2-substitued benzimidazoles [5-9]. A major drawback of these methods is the poor yields, use of expensive reagents and long reaction time. Moreover, economical and environmental conditions are forcing the chemical community to find more efficient ways for performing chemical reactions [10]. The use of microwave irradiation in chemical reactions leads to enhanced reaction rates, higher yields of products, easier work-up and, sometimes, to selective conversions with several advantages of the eco-friendly approach in the framework of green chemistry [11]. Consequently, this protocol should be welcome in these environmentally conscious days.

Because of these reasons, microwave-assisted synthesis of benzimidazoles has been come into prominence and many benzimidazole derivatives have been gained in literature. In this study, we wish to report the synthesis of 5-nitro- $1 H$-benzimidazoles and their hydrazide derivatives starting from iminoester hydrochlorides, which can be easily obtained, under microwave irradiation and with conventional heating.

\section{RESULTS AND DISCUSSION}

Synthesis of the compounds $\mathbf{4}(\mathbf{a}-\mathbf{d})$ has been carried out as depicted in Scheme 1. Ethylimidobenzylacetate hydrochlorides 1(a-d) was prepared according to the Pinner method [12-14]. To synthesize benzimidazole compounds, secondly, there has been developed a simple method using 4-nitro-o-phenylenediamine and ethylimidobenzylacetate hydrochlorides $\mathbf{1}(\mathbf{a}-\mathbf{d})$

*Corresponding author. E-mail: emre.mentese@erdogan.edu.tr 
with microwave irradiation and classical method, then, 5(6)-nitro- $1 H$-benzimidazoles 2(a-d) have been obtained. Ethyl [2-benzyl-5(6)-nitro-1H-benzimidazol-1-yl]acetates 3(a-d) have been prepared with treatment of compounds $2(\mathbf{a}-\mathbf{d})$ and ethyl bromoacetate in acetone. Finally, compounds 3(a-d) has been reacted with hydrazine monohydrate in ethanol to synthesize 2-[5(6)-nitro-2-benzyl)-1H-benzimidazol-1-yl]acetohydrazide 4(a-d). All reactions have been carried out with conventional heating and under microwave irradiation and results were compared (Table 1.).

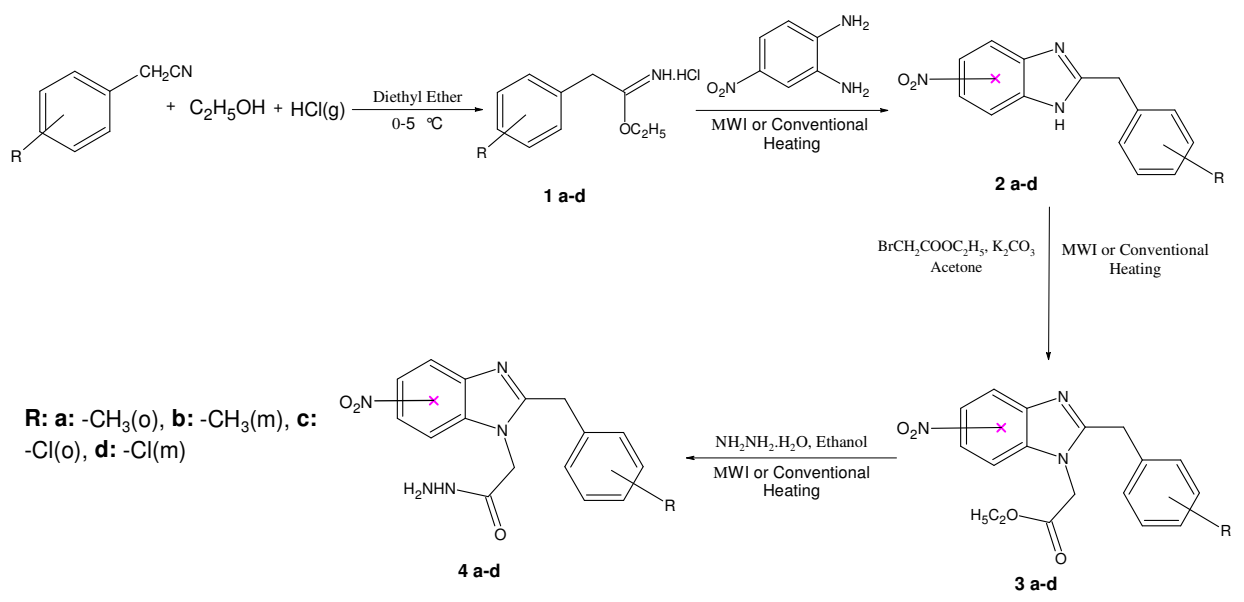

Scheme 1. Synthetic route for compounds $\mathbf{2 a - d , ~ 3 a - d ~ a n d ~ 4 a - d . ~}$

Table 1. Comparation of yields and reaction times of classical and microwave condition.

\begin{tabular}{|c|c|c|c|c|c|}
\hline \multirow{2}{*}{ Compound } & \multicolumn{3}{|c|}{ Classical conditions } & \multicolumn{3}{c|}{ Microwave conditions } \\
\cline { 2 - 6 } & Time $(\mathrm{h})$ & Yield $^{\mathrm{a}}(\%)$ & Temperature $\left({ }^{\circ} \mathrm{C}\right)$ & Time $(\mathrm{min})$ & Yield $^{\mathrm{a}}(\%)$ \\
\hline $\mathbf{2 a}$ & 6 & $71\left(60^{\mathrm{b}}[18]\right)$ & 60 & 10 & 87 \\
\hline $\mathbf{2 b}$ & 6 & $79\left(65^{\mathrm{b}}[18]\right)$ & 60 & 10 & 91 \\
\hline $\mathbf{2 c}$ & 6 & 80 & 60 & 10 & 93 \\
\hline $\mathbf{2 d}$ & 6 & 72 & 60 & 10 & 88 \\
\hline 3a & 10 & 86 & 90 & 15 & 96 \\
\hline 3b & 10 & 89 & 90 & 15 & 92 \\
\hline 3c & 10 & 79 & 90 & 15 & 95 \\
\hline 3d & 10 & 74 & 90 & 15 & 90 \\
\hline 4a & 6 & 50 & 120 & 10 & 79 \\
\hline $4 \mathbf{4 b}$ & 6 & 45 & 120 & 10 & 70 \\
\hline $4 \mathbf{4 c}$ & 6 & 54 & 120 & 10 & 71 \\
\hline $4 \mathbf{4 d}$ & 6 & 40 & 120 & 10 & 65 \\
\hline
\end{tabular}

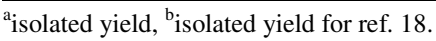

The IR spectrum of all compounds shows characteristic absorptions bands at about 1510 and $1315 \mathrm{~cm}^{-1}$ for $-\mathrm{NO}_{2}$ and $1620 \mathrm{~cm}^{-1}$ for $\mathrm{C}=\mathrm{N}$. The $\mathrm{C}=\mathrm{O}$ band is at about $1735 \mathrm{~cm}^{-1}$ and $1645 \mathrm{~cm}^{-1}$ for compounds 3(a-d) and 4(a-d), respectively. Also, compounds 4(a-d) have new $\mathrm{NH}$ and $\mathrm{NH}_{2}$ signals between $3300-3296 \mathrm{~cm}^{-1}$ because of hydrazide formation.

${ }^{1}$ H-NMR spectra of each compound shows compatible signals with proposed structures. $\mathrm{N}-\mathrm{H}$ signals are shown at about $13.00 \mathrm{ppm}$ for compounds $\mathbf{2}$ (a-d) and $9.75 \mathrm{ppm}$ for compounds 
for compounds $\mathbf{4}(\mathbf{a}-\mathbf{d})$. With the alkylation reaction of compounds $\mathbf{2}(\mathbf{a}-\mathbf{d})$, - $\mathrm{NH}$ signals have been left and new signals belonging to $-\mathrm{NCH}_{2} \mathrm{COOEt}$ have been shown for compounds 3(a-d). With the conversion of compounds $\mathbf{3}$ (a-d) to their hydrazide derivatives $\mathbf{4}(\mathbf{a}-\mathbf{d})$, the $\mathrm{OCH}_{2}$ and $-\mathrm{OCH}_{2} \mathrm{CH}_{3}$ signals disappeared and new $-\mathrm{NH}$ and $-\mathrm{NH}_{2}$ signals were shown.

${ }^{13} \mathrm{C}-\mathrm{NMR}$ spectra of each compounds showed compatible signals with proposed structures. $\mathrm{C}=\mathrm{O}$ signals are shown at about 175 and $168 \mathrm{ppm}$ for compounds 3(a-d) and 4(a-d), respectively. The signals belonging to $-\mathrm{OCH}_{2} \underline{\mathrm{CH}}_{3}$ group for compounds 3 (a-d) are at the related chemical values. $\mathrm{No}-\mathrm{OCH}_{2} \mathrm{CH}_{3}$ signals has been shown for compounds $\mathbf{4}(\mathbf{a}-\mathbf{d})$. That proves the hydrazide formation. In addition, all compounds gave stable $\mathrm{M}+1$ ion peak and reasonable elemental analysis data.

\section{EXPERIMENTAL}

All the chemicals were supplied from Merck, Aldrich and Fluka. Melting points were determined on capillary tubes on a Büchi oil heated melting point apparatus and uncorrected. ${ }^{1} \mathrm{H}$ - and ${ }^{13} \mathrm{C}$-NMR spectra were performed on Varian-Mercury $200 \mathrm{MHz}$ spectrophotometer in DMSO- $d_{6}$ using TMS as internal. The IR spectra were recorded on a Perkin-Elmer 100 FTIR spectrophotometer as $\mathrm{KBr}$ pellets. The elemental compositions were determined on a Carlo Erba $1106 \mathrm{CHN}$ analyzer; the experimental values were in agreement $( \pm 0.4 \%)$ with calculated ones. Mass spectra were recorded on Thermo Scientific Quantum Access max LC-MS spectrophotometer. A mono-mode CEM-Discover microwave was used to carry out microwave reactions in $30 \mathrm{~mL}$ microwave process vials with temperature control by infrared detection temperature sensor. All reactions were monitored by TLC using precoated aluminum sheets (silica gel 60 F $2.540 .2 \mathrm{~mm}$ thickness).

Synthesis of compounds 2 a-d

Conventional method. A mixture of 4-nitro-o-phenylenediamine $(0.01 \mathrm{~mol})$ and corresponding iminoester hydrochloride $\mathbf{1}(\mathbf{a}-\mathbf{d})(0.013 \mathrm{~mol})$ in methanol $(30 \mathrm{~mL})$ were stirred at room temperature for $4 \mathrm{~h}$. Then the mixture was refluxed for $2 \mathrm{~h}$. After the reaction was completed, monitored by TLC (EtAc:hexane, 3:1), the mixture was cooled down to room temperature, the product was precipitated with addition of water. The obtained product was filtered, dried and recrystallized from ethanol-water (1:1).

Microwave method. 4-Nitro-o-phenylenediamine $(0.01 \mathrm{~mol})$ and corresponding iminoester hydrochloride (1a-d) $(0.013 \mathrm{~mol})$ in methanol $(10 \mathrm{~mL})$ were taken in a closed vessel. Then, it was irradiated in microwave at $60{ }^{\circ} \mathrm{C}$ and $10 \mathrm{~min}$ (hold time) at 300 Watt maximum power. After the reaction was completed (monitored as stated above), the mixture was cooled down to room temperature and taken in a beaker. The product was precipitated by addition of water and purification methods mentioned above were applied.

2-(2-Methylbenzyl)-5(6)-nitro-1H-benzimidazole (2a). m.p.: $168-170{ }^{\circ} \mathrm{C}\left(165-166{ }^{\circ} \mathrm{C}[18]\right)$, IR $(\mathrm{KBr}): 3100(\mathrm{NH}), 3023(\mathrm{Ar}-\mathrm{H}), 1625(\mathrm{C}=\mathrm{N}), 1513,1322\left(\mathrm{NO}_{2}\right) \mathrm{cm}^{-1} .{ }^{1} \mathrm{H}-\mathrm{NMR}(200 \mathrm{MHz}$, DMSO- $_{6}$ ) $\delta: 12.95(\mathrm{NH}), 8.43$ (s, 1H, Ar-H), 8.12 (d, 1H, $\left.J=8.3 \mathrm{~Hz}, \mathrm{Ar}-\mathrm{H},\right), 8.03$ (d, 1H, $J=$ 8.3, Ar-H), 7.43-7.02 (m, 4H, Ar-H), $4.23\left(2 \mathrm{H}, \mathrm{t}, J=7.3, \mathrm{CH}_{2}\right), 2.27\left(3 \mathrm{H}, \mathrm{d}, J=7.3, \mathrm{CH}_{3}\right) .{ }^{13} \mathrm{C}-$ NMR (50 MHz, DMSO- $\left.d_{6}\right) \delta: 157.2(\mathrm{C}=\mathrm{N}), 145.3(\mathrm{C}), 143.1(\mathrm{C}), 140.9(\mathrm{C}), 136.7$ (C), 133.1 (C), $130.6(\mathrm{CH}), 128.5(2 \mathrm{CH}), 126.1(\mathrm{CH}), 124.3(2 \mathrm{CH}), 122.9(\mathrm{CH}), 32.7\left(\mathrm{CH}_{2}\right), 19.5\left(\mathrm{CH}_{3}\right)$. ESI-MS m/z (rel. int.) 268 [M+1] (100), 253 (37), 163 (51).

2-(3-Methylbenzyl)-5(6)-nitro-1H-benzimidazole (2b). m.p.: $170-171{ }^{\circ} \mathrm{C}\left(166-167{ }^{\circ} \mathrm{C}[18]\right)$, IR (KBr): $3094(\mathrm{NH}), 3009$ (Ar-H), $1623(\mathrm{C}=\mathrm{N}), 1513,1320\left(\mathrm{NO}_{2}\right) \mathrm{cm}^{-1}$. ${ }^{1} \mathrm{H}-\mathrm{NMR}(200 \mathrm{MHz}$, 
DMSO- $\left.d_{6}\right) \delta: 12.78(\mathrm{NH}), 8.49(\mathrm{~s}, 1 \mathrm{H}, \mathrm{Ar}-\mathrm{H}), 8.08(\mathrm{~d}, 1 \mathrm{H}, J=8.1 \mathrm{~Hz}, \mathrm{Ar}-\mathrm{H}),, 7.92(\mathrm{~d}, 1 \mathrm{H}, J=$ 8.1, Ar-H), 7.40-6.92 (m, 4H, Ar-H), $4.23\left(2 \mathrm{H}, \mathrm{s}, \mathrm{CH}_{2}\right), 2.27\left(3 \mathrm{H}, \mathrm{s}, \mathrm{CH}_{3}\right) .{ }^{13} \mathrm{C}-\mathrm{NMR}(50 \mathrm{MHz}$, DMSO- $\left.d_{6}\right) \delta: 159.2(\mathrm{C}=\mathrm{N}), 149.3(\mathrm{C}), 141.1(2 \mathrm{C}), 136.7(\mathrm{C}), 133.1(\mathrm{C}), 131.6(\mathrm{CH}), 128.5$ $(\mathrm{CH}), 128.0(\mathrm{CH}), 125.1(2 \mathrm{CH}), 123.0(\mathrm{CH}), 121.7(\mathrm{CH}), 34.1\left(\mathrm{CH}_{2}\right), 20.3\left(\mathrm{CH}_{3}\right)$. ESI-MS $\mathrm{m} / \mathrm{z}$ (rel. int.) 268 [M+1] (100), 253 (39), 163 (69).

2-(2-Chlorobenzyl)-5(6)-nitro-1H-benzimidazole (2c). m.p.: 203-204 ${ }^{\circ} \mathrm{C}$, IR (KBr): $3114(\mathrm{NH})$, 3018 (Ar-H), $1621(\mathrm{C}=\mathrm{N}), 1515,1327\left(\mathrm{NO}_{2}\right) \mathrm{cm}^{-1} .{ }^{1} \mathrm{H}-\mathrm{NMR}\left(200 \mathrm{MHz}, \mathrm{DMSO}-d_{6}\right) \delta: 13.05$ $(\mathrm{NH}), 8.37(\mathrm{~s}, 1 \mathrm{H}, \mathrm{Ar}-\mathrm{H}), 8.09$ (d, $1 \mathrm{H}, J=8.3 \mathrm{~Hz}, \mathrm{Ar}-\mathrm{H}), 7.90(\mathrm{~d}, 1 \mathrm{H}, J=8.3, \mathrm{Ar}-\mathrm{H}), 7.35-7.00$ $(\mathrm{m}, 4 \mathrm{H}, \mathrm{Ar}-\mathrm{H}), 4.39\left(2 \mathrm{H}, \mathrm{t}, J=7.3, \mathrm{CH}_{2}\right) .{ }^{13} \mathrm{C}-\mathrm{NMR}\left(50 \mathrm{MHz}, \mathrm{DMSO}-d_{6}\right) \delta: 155.1(\mathrm{C}=\mathrm{N})$, $149.2(\mathrm{C}), 145.2(\mathrm{C}), 141.9(\mathrm{C}), 136.7(2 \mathrm{C}), 131.2(\mathrm{CH}), 129.5(\mathrm{CH}), 125.3(2 \mathrm{CH}), 123.1(\mathrm{CH})$, $120.9(2 \mathrm{CH}), 36.7\left(\mathrm{CH}_{2}\right)$. ESI-MS m/z (rel. int.) 290 [M+3] (35), 288 [M+1] (100), 177 (47), 163 (51). Anal. C $58.45 \%$, H 3.50\%, N $14.61 \%$, calcd. for $\mathrm{C}_{14} \mathrm{H}_{10} \mathrm{ClN}_{3} \mathrm{O}_{2}, 58.41 \%, \mathrm{H} 3.52 \%$, N $14.64 \%$.

2-(3-Chlorobenzyl)-5(6)-nitro-1H-benzimidazole (2d). m.p.: 198-200 ${ }^{\circ} \mathrm{C}$, IR (KBr): $3110(\mathrm{NH})$, $3008(\mathrm{Ar}-\mathrm{H}), 1629(\mathrm{C}=\mathrm{N}), 1518,1320\left(\mathrm{NO}_{2}\right) \mathrm{cm}^{-1} .{ }^{1} \mathrm{H}-\mathrm{NMR}\left(200 \mathrm{MHz}, \mathrm{DMSO}-d_{6}\right) \delta: 13.00$ $(\mathrm{NH}), 8.30$ (s, 1H, Ar-H), 8.04 (d, 1H, J= 8.5 Hz, Ar-H,), 7.99 (d, 1H, J = 8.5, Ar-H), 7.21-6.00 $(\mathrm{m}, 4 \mathrm{H}, \mathrm{Ar}-\mathrm{H}), 4.41\left(2 \mathrm{H}, \mathrm{t}, J=7.3, \mathrm{CH}_{2}\right) .{ }^{13} \mathrm{C}-\mathrm{NMR}\left(50 \mathrm{MHz}, \mathrm{DMSO}-d_{6}\right) \delta: 153.2(\mathrm{C}=\mathrm{N})$, $148.1(\mathrm{C}), 145.9(\mathrm{C}), 146.0(\mathrm{C}), 138.7(\mathrm{C}), 136.2(\mathrm{C}), 135.1(\mathrm{CH}), 132.7(\mathrm{CH}), 129.3(2 \mathrm{CH})$, $125.1(\mathrm{CH}), 121.2(\mathrm{CH}), 199.1(\mathrm{CH}) 39.1\left(\mathrm{CH}_{2}\right)$. ESI-MS m/z (rel. int.) $290[\mathrm{M}+3](35), 288$ [M+1] (100), 177 (42), 163 (69). Anal. C 58.45\%, H 3.50\%, N $14.61 \%$, calcd. for $\mathrm{C}_{14} \mathrm{H}_{10} \mathrm{ClN}_{3} \mathrm{O}_{2}, 58.43 \%, \mathrm{H} 3.51 \%, \mathrm{~N} 14.59 \%$.

Synthesis of compounds $\mathbf{3 a - d}$

Conventional method. A mixture of compound 2a-d (0.01 mol), ethyl bromoacetate $(0.01 \mathrm{~mol})$ and dry $\mathrm{K}_{2} \mathrm{CO}_{3}(0.03 \mathrm{~mol})$ in acetone $(20 \mathrm{~mL})$ were stirred in a room temperature for $10 \mathrm{~h}$. After the reaction was completed, monitored by TLC (EtAc:hexane, 4:1), the product was precipitated by addition of water and was filtrated, dried and recrystallized from ethanol to afford the desired product.

Microwave method. A solution of compound 2a-d $(0.01 \mathrm{~mol})$ in acetone $(10 \mathrm{~mL})$ was taken in a closed vessel and dry $\mathrm{K}_{2} \mathrm{CO}_{3}(0.03 \mathrm{~mol})$ was added. The mixture was irritated in microwave at $90{ }^{\circ} \mathrm{C}, 5 \mathrm{~min}$ (hold time, 5 bar) at 300 Watt maximum power. Then, the mixture was cooled down to room temperature and ethyl bromoacetate $(0.01 \mathrm{~mol})$ was added. Again, it was irradiated in microwave at $90{ }^{\circ} \mathrm{C}$ and $10 \mathrm{~min}$ (hold time) at 300 Watt maximum power. After the reaction was completed (monitored as stated above), the mixture was cooled, taken in a beaker and the product was precipitated by addition of water. Purification methods mentioned above were applied to yield the pure product.

Ethyl [2-(2-methylbenzyl)-5(6)-nitro-1H-benzimidazol-1-yl] acetate (3a). m.p.: 130-132 ${ }^{\circ} \mathrm{C}$, IR $(\mathrm{KBr}): 3019(\mathrm{Ar}-\mathrm{H}), 1736(\mathrm{C}=\mathrm{O}), 1620(\mathrm{C}=\mathrm{N}), 1517,1320\left(\mathrm{NO}_{2}\right) \mathrm{cm}^{-1} .{ }^{1} \mathrm{H}-\mathrm{NMR}(200 \mathrm{MHz}$, DMSO- $\left.d_{6}\right) \delta: 8.50(\mathrm{~s}, 1 \mathrm{H}, \mathrm{Ar}-\mathrm{H}), 8.22(\mathrm{~d}, 1 \mathrm{H}, J=8.0 \mathrm{~Hz}, \mathrm{Ar}-\mathrm{H}),, 8.10(\mathrm{~d}, 1 \mathrm{H}, J=8.0, \mathrm{Ar}-\mathrm{H})$, 7.73-7.29 (m, 4H, Ar-H), $5.37\left(2 \mathrm{H}, \mathrm{s}, \mathrm{N}-\mathrm{CH}_{2}\right), 4.23\left(2 \mathrm{H}, \mathrm{q}, J=7.3, \mathrm{CH}_{2}\right), 4.00(2 \mathrm{H}, \mathrm{q}, J=7.2$ $\left.\mathrm{Hz}, \mathrm{OCH}_{2}\right), 2.27\left(3 \mathrm{H}, \mathrm{t}, J=7.3, \mathrm{CH}_{3}\right), 1.10\left(3 \mathrm{H}, \mathrm{t}, J=7.2, \mathrm{CH}_{3}\right) .{ }^{13} \mathrm{C}-\mathrm{NMR}(50 \mathrm{MHz}, \mathrm{DMSO}-$ $\left.d_{6}\right) \delta: 170.3(\mathrm{C}=\mathrm{O}), 157.6(\mathrm{C}=\mathrm{N}), 148.2(\mathrm{C}), 142.1(\mathrm{C}), 140.0(\mathrm{C}), 136.7(2 \mathrm{C}), 132.6(\mathrm{CH})$, $128.5(\mathrm{CH}), 127.7(\mathrm{CH}), 126.1(\mathrm{CH}), 124.3(\mathrm{CH}), 123.2(\mathrm{CH}), 122.9(\mathrm{CH}), 47.2\left(\mathrm{CH}_{2}\right), 32.7$ $\left(\mathrm{CH}_{2}\right), 19.5\left(\mathrm{CH}_{3}\right), 15.9\left(\mathrm{CH}_{3}\right)$.ESI-MS m/z (rel. int.) 354 [M+1] (100), 267 (65), 263 (34), 163 
Microwave-assisted synthesis of some 5(6)-nitro- $1 H$-benzimidazoles and their hydrazides 269

(39). Anal. C 64.58\%, H 5.42\%, N $11.89 \%$, calcd. for $\mathrm{C}_{19} \mathrm{H}_{19} \mathrm{~N}_{3} \mathrm{O}_{4}, \mathrm{C} 64.55 \%, \mathrm{H} 5.43 \%, \mathrm{~N}$ $11.91 \%$.

Ethyl [2-(3-methylbenzyl)-5(6)-nitro-1H-benzimidazol-1-yl] acetate (3b). m.p.: 140-141 ${ }^{\circ} \mathrm{C}$, IR $(\mathrm{KBr}): 3003(\mathrm{Ar}-\mathrm{H}), 1740(\mathrm{C}=\mathrm{O}), 1622(\mathrm{C}=\mathrm{N}), 1510,1329\left(\mathrm{NO}_{2}\right) \mathrm{cm}^{-1} .{ }^{1} \mathrm{H}-\mathrm{NMR}(200 \mathrm{MHz}$, DMSO- $\left.d_{6}\right) \delta: 8.61(\mathrm{~s}, 1 \mathrm{H}, \mathrm{Ar}-\mathrm{H}), 8.23(\mathrm{~d}, 1 \mathrm{H}, J=8.1 \mathrm{~Hz}, \mathrm{Ar}-\mathrm{H}),, 8.10(\mathrm{~d}, 1 \mathrm{H}, J=8.1, \mathrm{Ar}-\mathrm{H})$, 7.70-7.27 (m, 4H, Ar-H), $5.31\left(2 \mathrm{H}, \mathrm{s}, \mathrm{N}-\mathrm{CH}_{2}\right), 4.20\left(2 \mathrm{H}, \mathrm{s}, \mathrm{CH}_{2}\right), 4.11(2 \mathrm{H}, \mathrm{q}, J=7.0 \mathrm{~Hz}$, $\left.\mathrm{OCH}_{2}\right), 2.25\left(3 \mathrm{H}, \mathrm{s}, \mathrm{CH}_{3}\right), 1.10\left(3 \mathrm{H}, \mathrm{t}, J=7.0 \mathrm{~Hz}, \mathrm{CH}_{3}\right) .{ }^{13} \mathrm{C}-\mathrm{NMR}\left(50 \mathrm{MHz}, \mathrm{DMSO}-d_{6}\right) \delta$ : $172.1(\mathrm{C}=\mathrm{O}), 158.2(\mathrm{C}=\mathrm{N}), 147.1(\mathrm{C}), 142.8(\mathrm{C}), 140.5(\mathrm{C}), 139.1(2 \mathrm{C}), 135.2(\mathrm{CH}), 129.5$ $(\mathrm{CH}), 128.1(\mathrm{CH}), 125.2(\mathrm{CH}), 123.1(\mathrm{CH}), 120.1(\mathrm{CH}), 118.2(\mathrm{CH}), 45.2\left(\mathrm{CH}_{2}\right), 36.7\left(\mathrm{CH}_{2}\right)$, $19.5\left(\mathrm{CH}_{3}\right), 14.9\left(\mathrm{CH}_{3}\right)$. ESI-MS m/z (rel. int.) $354[\mathrm{M}+1]$ (100), 267 (55), $263(44), 163(43)$. Anal. C 64.58\%, H 5.42\%, N $11.89 \%$, calcd. for $\mathrm{C}_{19} \mathrm{H}_{19} \mathrm{~N}_{3} \mathrm{O}_{4}, \mathrm{C} 64.57 \%$, H 5.45\%, N $11.89 \%$.

Ethyl [2-(2-chlorobenzyl)-5(6)-nitro-1H-benzimidazol-1-yl] acetate (3c). m.p.: 125-127 ${ }^{\circ} \mathrm{C}$, IR (KBr): $3011(\mathrm{Ar}-\mathrm{H}), 1742(\mathrm{C}=\mathrm{O}), 1624(\mathrm{C}=\mathrm{N}), 1511,1319\left(\mathrm{NO}_{2}\right) \mathrm{cm}^{-1} .{ }^{1} \mathrm{H}-\mathrm{NMR}(200 \mathrm{MHz}$, DMSO- $\left.d_{6}\right) \delta: 8.60(\mathrm{~s}, 1 \mathrm{H}, \mathrm{Ar}-\mathrm{H}), 8.29(\mathrm{~d}, 1 \mathrm{H}, J=8.2 \mathrm{~Hz}, \mathrm{Ar}-\mathrm{H}),, 8.10(\mathrm{~d}, 1 \mathrm{H}, J=8.2, \mathrm{Ar}-\mathrm{H})$, 7.73-7.17 (m, $4 \mathrm{H}, \mathrm{Ar}-\mathrm{H}), 5.22\left(2 \mathrm{H}, \mathrm{s}, \mathrm{N}-\mathrm{CH}_{2}\right), 4.22\left(2 \mathrm{H}, \mathrm{s}, \mathrm{CH}_{2}\right), 4.16(2 \mathrm{H}, \mathrm{q}, J=7.0 \mathrm{~Hz}$, $\left.\mathrm{OCH}_{2}\right), 1.19\left(3 \mathrm{H}, \mathrm{t}, J=7.0 \mathrm{~Hz}, \mathrm{CH}_{3}\right) .{ }^{13} \mathrm{C}-\mathrm{NMR}\left(50 \mathrm{MHz}, \mathrm{DMSO}-d_{6}\right) \delta: 175.2(\mathrm{C}=\mathrm{O}), 159.2$ $(\mathrm{C}=\mathrm{N}), 145.9(\mathrm{C}), 143.1(\mathrm{C}), 141.8(\mathrm{C}), 138.1(\mathrm{C}), 136.4(\mathrm{C}), 134.7(\mathrm{CH}), 131.5(\mathrm{CH}), 129.1$ $(\mathrm{CH}), 125.2(\mathrm{CH}), 123.1(\mathrm{CH}), 120.1(\mathrm{CH}), 118.2(\mathrm{CH}), 45.2\left(\mathrm{CH}_{2}\right), 36.7\left(\mathrm{CH}_{2}\right), 14.9\left(\mathrm{CH}_{3}\right)$. ESI-MS m/z (rel. int.) 376 [M+3] (35), 374 [M+1] (100), 339 (32), 290 (13), 292 (40), 288(23), 267 (55), 163 (31). Anal. C 57.84\%, H 4.31\%, N $11.24 \%$, calcd. for $\mathrm{C}_{18} \mathrm{H}_{16} \mathrm{ClN}_{3} \mathrm{O}_{4}, \mathrm{C} 57.87 \%$, H $4.33 \%$, N $11.26 \%$.

Ethyl [2-(3-chlorobenzyl)-5(6)-nitro-1H-benzimidazol-1-yl] acetate (3d). m.p.: 115-116 ${ }^{\circ} \mathrm{C}$, IR (KBr): $3010(\mathrm{Ar}-\mathrm{H}), 1745(\mathrm{C}=\mathrm{O}), 1622(\mathrm{C}=\mathrm{N}), 1513,1318\left(\mathrm{NO}_{2}\right) \mathrm{cm}^{-1} .{ }^{1} \mathrm{H}-\mathrm{NMR}(200 \mathrm{MHz}$, DMSO- $\left.d_{6}\right) \delta: 8.63(\mathrm{~s}, 1 \mathrm{H}, \mathrm{Ar}-\mathrm{H}), 8.40(\mathrm{~d}, 1 \mathrm{H}, J=8.1 \mathrm{~Hz}, \mathrm{Ar}-\mathrm{H}),, 8.17(\mathrm{~d}, 1 \mathrm{H}, J=8.1, \mathrm{Ar}-\mathrm{H})$, 7.73-7.17 (m, 4H, Ar-H), $5.40\left(2 \mathrm{H}, \mathrm{s}, \mathrm{N}-\mathrm{CH}_{2}\right), 4.39\left(2 \mathrm{H}, \mathrm{s}, \mathrm{CH}_{2}\right), 4.19(2 \mathrm{H}, \mathrm{q}, J=7.1 \mathrm{~Hz}$, $\left.\mathrm{OCH}_{2}\right), 1.28\left(3 \mathrm{H}, \mathrm{t}, J=7.1 \mathrm{~Hz}, \mathrm{CH}_{3}\right) .{ }^{13} \mathrm{C}-\mathrm{NMR}\left(50 \mathrm{MHz}, \mathrm{DMSO}-d_{6}\right) \delta: 176.1(\mathrm{C}=\mathrm{O}), 158.9$ $(\mathrm{C}=\mathrm{N}), 147.7(\mathrm{C}), 145.1(\mathrm{C}), 143.8(\mathrm{C}), 139.9(\mathrm{C}), 137.8(\mathrm{C}), 136.2(\mathrm{CH}), 133.8(\mathrm{CH}), 130.0$ $(\mathrm{CH}), 127.9(\mathrm{CH}), 124.8(\mathrm{CH}), 122.5(\mathrm{CH}), 119.9(\mathrm{CH}), 44.7\left(\mathrm{CH}_{2}\right), 38.0\left(\mathrm{CH}_{2}\right), 15.6\left(\mathrm{CH}_{3}\right)$. ESI-MS m/z (rel. int.) 376 [M+3] (35), 374 [M+1] (100), 339 (40), 290 (20), 292 (58), 288 (36) 267 (55), 163 (31). Anal. C 57.84\%, H 4.31\%, N $11.24 \%$, calcd. for $\mathrm{C}_{18} \mathrm{H}_{16} \mathrm{ClN}_{3} \mathrm{O}_{4}, \mathrm{C} 57.87 \%$, H $4.33 \%$, N $11.26 \%$.

Synthesis of 2-(2-alkyl/aryl-5(6)-nitro-1H-benzimidazol-1-il)acetohydrazide (4a-d)

Conventional method. A mixture of 3a-d $(0.01 \mathrm{~mol})$ and hydrazine monohydrate $(0.02 \mathrm{~mol})$ in ethanol $(30 \mathrm{~mL})$ was refluxed for $6 \mathrm{~h}$. After the reaction was completed, monitored by TLC (ethyl acetate:hexane, 3:1), the mixture was cooled down to room temperature and a white solid appeared. This crude product was filtrated, dried and recrystallized from ethanol.

Microwave method. A solution of 3a-d $(0.01 \mathrm{~mol})$ in ethanol $(10 \mathrm{~mL})$ and hydrazine monohydrate $(0.02 \mathrm{~mol})$ were taken in a closed vessel. The mixture was irradiated in microwave at $120^{\circ} \mathrm{C}$ and $10 \mathrm{~min}$ (hold time, $7 \mathrm{bar}$ ) at 300 Watt maximum power. After the reaction was completed (monitored as stated above), the mixture was cooled down to room temperature, taken in a beaker and a white solid appeared. This crude product was filtrated and purification methods mentioned above were applied. 
2-[2-(2-Methylbenzyl)-5(6)-nitro-1H-benzimidazol-1-yl] acetohydrazide (4a). m.p.: 250-252 ${ }^{\circ} \mathrm{C}$, IR (KBr): $3298\left(\mathrm{NH}, \mathrm{NH}_{2}\right), 3049(\mathrm{Ar}-\mathrm{H}), 1656(\mathrm{C}=\mathrm{O}), 1610(\mathrm{C}=\mathrm{N}), 1513,1315\left(\mathrm{NO}_{2}\right) \mathrm{cm}^{-1} .{ }^{1} \mathrm{H}-$ NMR (200 MHz, DMSO-d $\left.d_{6}\right) \delta: 9.65$ (s, 1H, NH), $8.44(\mathrm{~s}, 1 \mathrm{H}, \mathrm{Ar}-\mathrm{H}), 8.25(\mathrm{~d}, 1 \mathrm{H}, J=8.1 \mathrm{~Hz}$, Ar-H), $8.13(\mathrm{~d}, 1 \mathrm{H}, J=8.1, \mathrm{Ar}-\mathrm{H}), 7.83-7.19(\mathrm{~m}, 4 \mathrm{H}, \mathrm{Ar}-\mathrm{H}), 4.92\left(2 \mathrm{H}, \mathrm{s}, \mathrm{N}-\mathrm{CH}_{2}\right), 4.36(2 \mathrm{H}, \mathrm{s}$, $\left.\mathrm{NH}_{2}\right), 4.17\left(2 \mathrm{H}, \mathrm{q}, J=7.2, \mathrm{CH}_{2}\right), 2.27\left(3 \mathrm{H}, \mathrm{t}, J=7.2, \mathrm{CH}_{3}\right) .{ }^{13} \mathrm{C}-\mathrm{NMR}\left(50 \mathrm{MHz}, \mathrm{DMSO}-d_{6}\right) \delta$ : $165.3(\mathrm{C}=\mathrm{O}), 158.1(\mathrm{C}=\mathrm{N}), 149.1(\mathrm{C}), 144.2(\mathrm{C}), 142.6(\mathrm{C}), 137.3(\mathrm{C}), 135.4,133.7(\mathrm{CH})$, $129.2(\mathrm{CH}), 127.7(\mathrm{CH}), 126.9(\mathrm{CH}), 122.9(\mathrm{CH}), 120.1(\mathrm{CH}), 118.5(\mathrm{CH}), 50.2\left(\mathrm{CH}_{2}\right), 35.3$ $\left(\mathrm{CH}_{2}\right), 19.5\left(\mathrm{CH}_{3}\right)$. ESI-MS m/z (rel. int.) 340 [M+1] (100), 305 (26), 267 (52), 235 (34). Anal. C $60.17 \%$, H 5.05\%, N $20.64 \%$, calcd. for $\mathrm{C}_{17} \mathrm{H}_{17} \mathrm{~N}_{5} \mathrm{O}_{3}, \mathrm{C} 60.13 \%, \mathrm{H} 5.05 \%$, N $20.65 \%$.

2-[2-(3-Methylbenzyl)-5(6)-nitro-1H-benzimidazol-1-yl]acetohydrazide (4b). m.p.: 255-256 ${ }^{\circ} \mathrm{C}$, IR (KBr): $3299\left(\mathrm{NH}, \mathrm{NH}_{2}\right), 3032(\mathrm{Ar}-\mathrm{H}), 1654(\mathrm{C}=\mathrm{O}), 1619(\mathrm{C}=\mathrm{N}), 1510,1312\left(\mathrm{NO}_{2}\right) \mathrm{cm}^{-1} .{ }^{1} \mathrm{H}-$ NMR $\left(200 \mathrm{MHz}, \mathrm{DMSO}-d_{6}\right) \delta: 9.75(\mathrm{~s}, 1 \mathrm{H}, \mathrm{NH}), 8.52(\mathrm{~s}, 1 \mathrm{H}, \mathrm{Ar}-\mathrm{H}), 8.31(\mathrm{~d}, 1 \mathrm{H}, J=8.0 \mathrm{~Hz}$, $\mathrm{Ar}-\mathrm{H},), 8.18(\mathrm{~d}, 1 \mathrm{H}, J=8.0, \mathrm{Ar}-\mathrm{H}), 7.91-7.23(\mathrm{~m}, 4 \mathrm{H}, \mathrm{Ar}-\mathrm{H}), 4.89\left(2 \mathrm{H}, \mathrm{s}, \mathrm{N}-\mathrm{CH}_{2}\right), 4.32(2 \mathrm{H}, \mathrm{s}$, $\left.\mathrm{NH}_{2}\right), 4.14\left(2 \mathrm{H}, \mathrm{s}, \mathrm{CH}_{2}\right), 2.27\left(3 \mathrm{H}, \mathrm{s}, \mathrm{CH}_{3}\right) .{ }^{13} \mathrm{C}-\mathrm{NMR}\left(50 \mathrm{MHz}, \mathrm{DMSO}-d_{6}\right) \delta: 167.1(\mathrm{C}=\mathrm{O})$, $159.2(\mathrm{C}=\mathrm{N}), 150.3(\mathrm{C}), 146.3(\mathrm{C}), 144.7(\mathrm{C}), 139.2(\mathrm{C}), 137.3,133.5(\mathrm{CH}), 129.1(\mathrm{CH}), 125.3$ $(\mathrm{CH}), 122.4(\mathrm{CH}), 121.4(\mathrm{CH}), 120.4(\mathrm{CH}), 117.3(\mathrm{CH}), 48.2\left(\mathrm{CH}_{2}\right), 33.3\left(\mathrm{CH}_{2}\right), 17.9\left(\mathrm{CH}_{3}\right)$. ESI-MS m/z (rel. int.) $340[\mathrm{M}+1]$ (100), 305 (29), 267 (57), 235 (63). Anal. C 60.17\%, H $5.05 \%$, N $20.64 \%$, calcd. for $\mathrm{C}_{17} \mathrm{H}_{17} \mathrm{~N}_{5} \mathrm{O}_{3}, \mathrm{C} 60.15 \%, \mathrm{H} 5.02 \%, \mathrm{~N} 20.67 \%$.

2-[2-(2-Chlorobenzyl)-5(6)-nitro-1H-benzimidazol-1-yl]acetohydrazide (4c). m.p.: 245-246 ${ }^{\circ} \mathrm{C}$, IR (KBr): $3309\left(\mathrm{NH}, \mathrm{NH}_{2}\right), 3012(\mathrm{Ar}-\mathrm{H}), 1657(\mathrm{C}=\mathrm{O}), 1617(\mathrm{C}=\mathrm{N}), 1511,1310\left(\mathrm{NO}_{2}\right) \mathrm{cm}^{-1} .{ }^{1} \mathrm{H}-$ NMR (200 MHz, DMSO- $\left.d_{6}\right) \delta: 9.89(\mathrm{~s}, 1 \mathrm{H}, \mathrm{NH}), 8.58(\mathrm{~s}, 1 \mathrm{H}, \mathrm{Ar}-\mathrm{H}), 8.38(\mathrm{~d}, 1 \mathrm{H}, J=8.1 \mathrm{~Hz}$, Ar-H), 8.18 (d, 1H, J = 8.1, Ar-H), 7.91-7.20 (m, 4H, Ar-H), $4.93\left(2 \mathrm{H}, \mathrm{s}, \mathrm{N}-\mathrm{CH}_{2}\right), 4.30(2 \mathrm{H}, \mathrm{s}$, $\left.\mathrm{NH}_{2}\right), 4.11\left(2 \mathrm{H}, \mathrm{s}, \mathrm{CH}_{2}\right) .{ }^{13} \mathrm{C}-\mathrm{NMR}\left(50 \mathrm{MHz}, \mathrm{DMSO}-d_{6}\right) \delta: 168.1(\mathrm{C}=\mathrm{O}), 160.2(\mathrm{C}=\mathrm{N}), 151.3$ (C), $147.3(\mathrm{C}), 145.7(\mathrm{C}), 140.2(\mathrm{C}), 137.9,133.1(\mathrm{CH}), 129.6(\mathrm{CH}), 125.4(\mathrm{CH}), 122.8(\mathrm{CH})$, $121.1(\mathrm{CH}), 120.4(\mathrm{CH}), 117.3(\mathrm{CH}), 48.2\left(\mathrm{CH}_{2}\right), 36.0\left(\mathrm{CH}_{2}\right)$. ESI-MS $m / z$ (rel. int.) $362[\mathrm{M}+3]$ (35), $360[\mathrm{M}+1]$ (100), 325 (50), 295 (9) 293 (29), 177 (32). Anal. C 53.42\%, H 3.92\%, N $19.47 \% \%$, calcd. for $\mathrm{C}_{16} \mathrm{H}_{14} \mathrm{ClN}_{5} \mathrm{O}_{3}, \mathrm{C} 53.41 \%, \mathrm{H} 3.93 \%$, N $19.45 \%$.

2-[2-(3-Chlorobenzyl)-5(6)-nitro-1H-benzimidazol-1-yl]acetohydrazide (4d). m.p.: 250-251 ${ }^{\circ} \mathrm{C}$, IR (KBr): $3300\left(\mathrm{NH}, \mathrm{NH}_{2}\right), 3009(\mathrm{Ar}-\mathrm{H}), 1655(\mathrm{C}=\mathrm{O}), 1610(\mathrm{C}=\mathrm{N}), 1511,1313\left(\mathrm{NO}_{2}\right) \mathrm{cm}^{-1} .{ }^{1} \mathrm{H}-$ NMR (200 MHz, DMSO-d $\left.)_{6}\right) \delta: 9.81(\mathrm{~s}, 1 \mathrm{H}, \mathrm{NH}), 8.60(\mathrm{~s}, 1 \mathrm{H}, \mathrm{Ar}-\mathrm{H}), 8.28(\mathrm{~d}, 1 \mathrm{H}, J=8.0 \mathrm{~Hz}$, Ar-H, ), 8.18 (d, 1H, $J=8.0$, Ar-H), 7.71-7.10 (m, 4H, Ar-H), $4.96\left(2 \mathrm{H}, \mathrm{s}, \mathrm{N}-\mathrm{CH}_{2}\right), 4.41(2 \mathrm{H}, \mathrm{s}$, $\left.\mathrm{NH}_{2}\right), 4.23\left(2 \mathrm{H}, \mathrm{s}, \mathrm{CH}_{2}\right) .{ }^{13} \mathrm{C}-\mathrm{NMR}\left(50 \mathrm{MHz}, \mathrm{DMSO}-d_{6}\right) \delta: 170.6(\mathrm{C}=\mathrm{O}), 161.3(\mathrm{C}=\mathrm{N}), 151.9$ $(\mathrm{C}), 148.9(\mathrm{C}), 146.0(\mathrm{C}), 143.2(\mathrm{C}), 138.9,135.9(\mathrm{CH}), 132.6(\mathrm{CH}), 128.4(\mathrm{CH}), 125.0(\mathrm{CH})$, $121.8(\mathrm{CH}), 120.0(\mathrm{CH}), 115.9(\mathrm{CH}), 51.3\left(\mathrm{CH}_{2}\right), 37.4\left(\mathrm{CH}_{2}\right)$. ESI-MS $m / z$ (rel. int.) $362[\mathrm{M}+3]$ (35), 360 [M+1] (100), 325 (41), 295 (13) 293 (20), 177 (52). Anal. C 53.42\%, H 3.92\%, N $19.47 \% \%$, calcd. for $\mathrm{C}_{16} \mathrm{H}_{14} \mathrm{ClN}_{5} \mathrm{O}_{3}, \mathrm{C} 53.44 \%, \mathrm{H} 3.90 \%, \mathrm{~N} 19.49 \%$.

\section{ACKNOWLEDGEMENT}

This study was funded by the Scientific and Technological Research Council of Turkey (TUBITAK, project no: 108T356).

\section{REFERENCES}

1. Sierra-Zenteno, A.; Galán-Vidal, C.; Tapia-Benavides, R. J. Mex. Chem. Soc. 2002, 46, 125.

2. Küçükbay, H.; Yılmaz, Ü.; Şireci, N.; Önganer, A.N. Turk. J. Chem. 2011, 35, 561. 
Microwave-assisted synthesis of some 5(6)-nitro- $1 H$-benzimidazoles and their hydrazides 271

3. Yalçın, İ.; Şener, E.; Özden, T.; Özden, S.; Akın, A. Eur. J. Med. Chem. 1990, 25, 705.

4. Alamgir, M.; Black, D.S.C.; Kumar, N. Top. Heterocycl. Chem. 2007, 9, 87.

5. Reddy, A.C.S.; Rao, P.S.; Venkataratnam, R.V. Tetrahedron Lett. 1996, 37, 2845.

6. Boufatah, N.; Gellis, A.; Maldonado, J.; Vanelle P. Tetrahedron 2004, 41, 9131.

7. Khurana, J.M.; Vij, S.; Vij, K. Synth. Commun. 2012, 42, 2606.

8. Dawood, K.M.; Abdel-Wahab B.F. Arkivoc 2010, 333.

9. Dawood, K.M.; Elvan, N.M.; Abdel-Wahab B. F. Arkivoc 2011, 111.

10. Varma, R.S. Microwave in Organic Synthesis, Wiley-VCH: Weinheim; 2012; Chap. 6, pp. 181.

11. Kappe, C.O. Angew. Chem. Int. Ed. 2004, 43, 6250.

12. Pinner, A. Die Imidoäther und ihre Derivate, 1 Auflage; Oppenheim: Berlin; 1892.

13. Kahveci, B. Molecules 2005, 10, 376.

14. İkizler, A.A.; Ün, R. Chim. Acta Turc. 1979, 7, 269.

15. Desai, K.G.; Desai, K.R. Bioorg. Med. Chem. 2006, 14, 8271.

16. Gowda, J.; Khadar, A.M.A.; Kumari, B.K.; Kumari, N.S. Indian J. Chem. 2010, 49, 1130.

17. Salman, A.S.S. J. Am. Sci. 2011, 7, 625.

18. Hunger, A.; Kebrle, J.; Rossi, A.; Hoffmann, K. Helv. Chim. Acta 1960, 43, 1032. 